MEDICAL PRACTICE

\title{
A case of reticulosis
}

\author{
STUART CARNE, MICHAEL WHITEHOUSE
}

British Medical fournal, 1977, 1, 492-494

\section{The trainee's problem}

The patient is a 10-year-old boy who attended the surgery two months ago complaining of a sore throat. He had an exudative tonsillitis with bilateral cervical lymphadenopathy, including glands in both anterior and posterior triangles. The left supraclavicular node was the most prominent, measuring about $2 \times 1.5 \mathrm{~cm}$, being soft and mobile but not tender. There was no hepatosplenomegaly and no other lymphadenopathy apart from some "scraggy" inguinal nodes. He was treated with penicillin V $250 \mathrm{mg}$ four times a day for seven days and was asked to return in a fortnight.

He was not seen again, however, until six weeks later, when he again attended with his mother, who is an ex-district nurse. $\mathrm{He}$ said that his throat was not troubling him, and he felt well. But his mother expressed concern about the left supraclavicular node, which was still enlarged and was occasionally painful, and posed the question ". . . could it be Hodgkin's disease . . . ??" The boy looked healthy, and examination showed nothing abnormal except for a persisting cervical lymphadenopathy with the left supraclavicular node being the most prominent. It had not changed in size but appeared to be more tender. There was again no hepatosplenomegaly, there were no enlarged axillary nodes, and the inguinal nodes were as previously noted. The boy appeared quite fit now and was coping well at school both academically and at sports. He is growing normally.

The problem, as I see it, is whether this node requires a

\section{London W12}

STUART CARNE, MB, FRCGP, general practitioner

Department of Medical Oncology, St Bartholomew's Hospital, London EC1A 7BE

J MICHAEL WHITEHOUSE, MD, MRCP, consultant physician and acting director biopsy and histological examination. The boy has had many upper respiratory tract infections in the past, and this in itself could explain his cervical lymphadenopathy, although it would be unusual for the one particular node to be so affected. I think, however, that now that the question of reticulosis has been raised confident reassurance cannot be given to his mother without a biopsy. My concern is how to balance my anxieties to protect this child (who I feel certain does not have Hodgkin's disease and, if he is anything like I was as a child, might find hospital frightening) from unnecessary and uncomfortable investigation, with the awareness that the mother is clearly not impressed by the opinion of a young doctor still in training.

\section{General practitioner's comments}

This case poses two problems that the general practitioner will have to solve to make his total assessment of the family's problems. Why is the left supraclavicular node enlarged; and why is the mother worried that her son may have Hodgkin's disease?

The reasons for an enlarged gland at this site in a 10-year-old boy may be considered under two main headings-infective and neoplastic. The most likely explanation for cervical adenitis is a chronic or recurrent upper respiratory tract infection, sometimes, but not always, related to a tonsillar infection. If the tonsils are infected, however, it would be more usual for the main adenopathy to be in the upper third of the anterior cervical chain.

Tuberculous cervical adenopathy must also not be forgotten, though it is uncommon these days except among some immigrants. A tuberculin test will help to exclude this possibility and most certainly should be part of the general practitioner's diagnostic work-up, though sometimes the diagnosis of tuberculous adenitis may have to rest on the results of a biopsy. Other rare infections may also have to be considered; the white cell count and sedimentation rate will indicate whether or not further investigations in that direction are warranted. A chronic dental infection and other types of sepsis in the mouth are also possibilities. The latter may be associated with leukaemia; the 
combination of oral sepsis and enlarged glands should make you think of this possibility.

Glandular fever does occur at this age and often the enlarged glands persist. The acute phase of mononucleosis often resembles a follicular tonsillitis. If a patient with tonsillitis fails to respond rapidly to penicillin I always ask for a white cell count and Paul-Bunnell test, as well as nose and throat swab cultures. I am impressed with how often organisms, usually staphylococci, can be cultured only from the nose in what appears to be a throat infection. I also look for the occasional patient who has glandular fever and a coincidental pharyngeal infection. The boy's story certainly fits in with mononucleosis. If the first consultation coincided with the onset of the episode the Paul-Bennell test should still be positive.

A negative throat swab does not rule out an infection. We see a lot of virus and other "non-specific" upper respiratory infections, particularly in children; many of them apparently allergic. The diagnosis may have to rest on the absence of other factors, though in practice one often makes this kind of diagnosis on the history and clinical findings-mainly the general wellbeing of the child confirmed, if necessary, by a few simple investigations.

\section{NEOPLASMS}

Neoplasms are rare in children, but there is a sort of perverse law which says it is in just such a case as this boy's that the general practitioner will meet this type of disease. He must, therefore, be particularly on his guard. Should the mother be right in her fears and should the general practitioner in any way seem to her to have delayed in getting treatment, then he will never be forgiven. Of the malignant diseases presenting in this way in a boy aged 10, leukaemia and Hodgkin's disease are the most likely but other reticuloses are also possible. The diagnosis of the latter will, however, be made as part of the same diagnostic process used for the first two possibilities.

What are the clinical features of Hodgkin's disease ? Firstly, it is uncommon at this age: it is more usually found in adults. Secondly, there are usually-but not always-features present which seem to be absent in this boy-weight loss, anorexia, anaemia, and, often, a periodic Pel-Ebstein fever. In other words, the child looks ill. Splenomegaly is present in only half the affected patients, so its absence in this boy is not of much importance.

The clinical picture is also not typical of the acute leukaemias seen in children. These children are usually anaemic and look it. Often there are haemorrhages owing to thrombocytopenia, manifesting as ecchymoses and purpura. Nevertheless, this diagnosis is just possible. The fact that acute leukaemia is common between the ages of 2 and 4 years is not helpful in making a diagnosis about one individual case. The cervical lymph glands are not the usual site for a Burkitt's tumour nor of sarcoid, though both conditions may have to be excluded if necessary.

There are all sorts of interpretations which may be placed on the mother's failure to bring her boy back to the doctor two weeks after the initial consultation as she was asked to do. This is not necessarily a sign of fecklessness, and it is as common a phenomenon among professional families as it is among others. Much more important is why did she bring up the question of Hodgkin's disease? She is a State-registered nurse and some nurses, like many doctors, always fear the worst. (A little learning. ...) Nevertheless, it does provide an excellent opportunity of re-establishing oneself in the eyes of this mother as a competent doctor and also, incidentally, such an approach may help in the future management of what is obviously a difficult family.

\section{SUGGESTED APPROACH}

A complete examination should be carried out, and this includes taking a full history. This will give the mother an opportunity to talk in her own words about her fears and problems in general. Don't interrupt, however irrelevant what she is saying may seem, and, of course, the examination must be fairly complete. I say "fairly" because I don't think a rectal examination, for example, is justified unless some other features appear in the history. Fundoscopy is, however, important, and tell the mother you are looking at the optic discs or she may think you want to see if the boy just needs glasses.

Even if a full history has already been obtained by asking many questions, the opportunity should be taken of saying to the mother that more details are wanted. The importance of taking a full history in this way was emphasised by Michael Balint when he said: "If you ask questions you get answers: if you want facts you have to listen."

It may be that after this examination of the position from a social and psychological angle as well as a clinical one the problem will become a lot clearer. If not, further investigations are indicated. A throat and nose swab, full blood count, ESR, and Paul-Bunnell tests are good for starters and a TB test (but first check to see if the boy has had a BCG in the past). My preference is for the TB tine test. This is more expensive than the Mantoux or Heaf but, being disposable, is easier to use, and one only needs to do it relatively infrequently in general practice. An $x$-ray examination of the chest may also be desirable, depending on the history.

What will probably happen is that these tests come back as normal. What then ? If the mother is still anxious, then I think the boy must be referred to a specialist. Indeed, I am a little worried about this gland myself. A supraclavicular node is less likely to be benign than a gland higher up in the cervical chain and I think I, too, would want a second opinion. But suppose this is one of the many instances when we are satisfied in our own minds that the lump is benign? The mother may still reject our opinion, though she will accept the same opinion from a specialist, and only then will she be happy to leave well alone. This evaluation of our respective capabilities by patients may be frustrating in one's early years in general practice, but once you learn to live with it there are times when you also can take advantage of the situation.

If the mother-or the doctor-still suspects that the boy may have Hodgkin's disease, there is only one diagnostic test, a biopsy, and this will have to be done. It will also be the final diagnostic arbiter should the boy have one of the other rare possibilities. And only a negative biopsy result will satisfy some parents (and also some doctors).

It you take your history properly, do the correct (the relatively simple) tests, and refer her sympathetically to a specialist, when the negative results come through she will be even more impressed with the fact that you were right-because you also did the right things.

\section{Consultant's comments}

The boy's history of sore throat and cervical lymphadenopathy is unremarkable but for one feature, and that is the persistence of left supraclavicular lymphadenopathy. Cervical lymphadenopathy is a common accompaniment of upper respiratory tract infection and usually disappears with resolution of the infection. Solitary supraclavicular lymphadenopathy is suggestive of mediastinal, testicular, or abdominal disease. While this may be infective in origin, solitary persistant lymphadenopathy of appreciable size is an uncommon presentation of infection apart from tubercle and, rarely, such conditions as toxoplasmosis or toxocara. It would appear from the history that, although upper respiratory tract infections have been common, they have cleared rapidly with appropriate antibiotic treatment. Other clinical findings have been unremarkable: neither the liver nor spleen could be felt.

Further questioning should be directed to determining whether there are any specific symptoms to suggest tuberculous infection-cough, weight loss and fever; intra-abdominal 
disease (Wilms's tumour or neuroblastoma)-abdominal discomfort, weight loss, fever, diarrhoea, or abdominal distension; and, finally, testicular swelling or pain. Unlike the trainee, I think Hodgkin's disease is a real possibility. Specific questions would be to determine whether there had been weight loss or night sweats. Occasionally pruritus occurs.

Hodgkin's disease is very rare before the age of 5 , but has a peak incidence between 8 and 11. It is three times more common in boys; and of the four kinds of histological appearances recognised as Hodgkin's disease-lymphocyte predominant, nodular sclerosing, mixed cellularity, and lymphocyte depletion -only the first three occur often in childhood. Painless lymphadenopathy in the lower cervical region, with or without symptoms, is the commonest presentation of Hodgkin's disease in children. In about half of the children with cervical lymphadenopathy and particularly those with nodular sclerosing histology the mediastinum is also involved.

So far as this boy is concerned, it is important that a diagnosis is made promptly. Indeed, the presence of a large lymph node for which no explanation can be found after six weeks is an indication for biopsy. The mother is moderately well informed and will not cease to be anxious until Hodgkin's disease is excluded. Her anxiety is likely to be transmitted to the child and the longer the delay in initiating investigations the greater the risk of his being infected with her anxiety. A careful examination should be carried out to exclude axillary, para-aortic, or appreciable inguinal lymphadenopathy and also to exclude hepatosplenomegaly. An $x$-ray examination of the chest (including penetrated and lateral views) and a Mantoux test will help to exclude mediastinal lymphadenopathy and pulmonary tuberculosis, but I think that a lymph node biopsy is unavoidable. This need not mean more than one night in hospital and will thus be minimally traumatic. Histological processing takes several days so that he can go straight home and come back to hospital for the stitches to be removed. If the lymph node histology is that of Hodgkin's disease, further assessment is essential.

\section{TREATMENT}

Since Hodgkin's disease spreads to contiguous lymph node groups, it is customary to irradiate disease confined to lymph nodes above the diaphragm and to include all other lymph node groups in the radiation field. This radiotherapy is given to the "mantle" area above the diaphragm and includes the mediastinum, axillae, and cervical, supraclavicular, and occipital lymph nodes. When lymph nodes below the diaphragm are affected, the para-aortic, iliac, inguinal, and femoral nodes are irradiated-the so-called inverted "Y" area. When there is disease confined to the lymph nodes both above the below the diaphragm, radiotherapy is given to both the mantle and inverted $\mathrm{Y}$ areas. Lymphocytic predominant and nodular sclerosing Hodgkin's disease are often fairly localised at presentation and confined to the lymph nodes, whereas mixed cellularity Hodgkin's disease may be much more extensive and include the bone marrow and liver.

Radiotherapy alone can cure localised disease-that is, that confined to the lymph nodes-so that the precise assessment of the extent of the disease is an essential prerequisite of treatment. In this boy's case the disease is likely to be confined to the lymph nodes and although lymphangiography may indicate intraabdominal involvement, its accuracy is not complete (less than $79 \%$ ). As radiotherapy, to be curative, depends on precise disease definition, laparotomy is necessary unless extranodal disease can be shown at the outset. At operation, samples of lymph nodes are required from all regions, particularly those that appear suspicious on a lymphangiogram or are obviously enlarged. The spleen is a notoriously difficult organ to assess. In adults half of those having a normal-size spleen are diseased and half of those with enlarged spleens have no evidence of splenic disease histologically. Furthermore, as the spleen overlies the upper pole of the left kidney this organ may be irradiated as a result of splenic irradiation, and radiation damage to the kidney may occur. Splenectomy in children is associated with a high incidence of postoperative infective complications, usually pneumococcal, and most centres prefer to avoid laparotomy and splenectomy in very young children. Prophylactic penicillin can completely eliminate the risk of bacterial infection, but even in children of this boy's age, the risk of postoperative infection cannot be ignored.

Chemotherapy has revolutionised the outlook of those patients with disseminated disease beyond the curative capacity of radiotherapy. This group of patients had a $10^{\circ}{ }_{0}$ three-year survival in the 1940 s, but now $80 \%$ achieve complete remission and survive five years or more. The major advance has come from the use of combination chemotherapy using mustine, vinblastine or vincristine, procarbazine, and prednisolone. Continuing chemotherapy for longer than the nine months required to give six courses of complete treatment is unnecessary in patients who achieve complete remission, and those who remain disease-free for two years or more stand an excellent chance of being cured.

Inevitably, the investigation and treatment of a potentially serious condition will cause particular anxiety to the parent of a young child. Modern management reduces the need for prolonged inpatient investigation, and indeed should the boy prove to have Hodgkin's disease the outlook is sufficiently good for all concerned-namely, general practitioner and specialist-to sustain his mother with critical optimism. In order to do so, however, it is absolutely essential that he is referred to a specialist centre with particular experience of the management of malignant disease in childhood. The problems of treatment are few but it must be explained to his mother that if chemotherapy is necessary the boy will almost certainly become sterile. Radiotherapy in the doses given in Britain should not affect his growth.

\section{Postscript to the problem}

A patient was referred to a haematologist and a biposy was performed a week later. The histology was reported as reactive hyperplasia and the next series of consultations was related to postoperative wound infection. No positive serological abnormalities have been found, and the blood indices are normal. Four months after the biopsy small glands were still palpable, and the consultant decided that the child should be seen again in six months' time "just to be sure." By then it will be more than 12 months since the first consultation. ...

It is not uncommon to read in the lay press of an apparently normal man physically who has undergone a "change of sex" operation. What advice is given about the growth of facial hair?

Once the pattern of facial hair growth has been established in the endocrinologically normal adult male it is almost impossible to stop it completely. Oestrogen will oppose the action of androgen on hair growth and an antiandrogen (such as cyproterone acetate) will also help. These measures are necessary because, while the castration that is carried out in a sex-change operation removes the main source of androgen-namely, the testes-there is still an appreciable contribution coming from the adrenal. Adrenal androgens maintain some of the male secondary sexual characteristics normally supported by testicular androgens. In particular, the hair follicles of the face, once programmed to produce a stubbly growth of beard, tend to continue this unless the recommended treatment is employed. On the other hand some "sex-change" operations are carried out in patients who are already suffering from clinical abnormalities of sexual differentiation and development. In many of these the problems over facial hair are not nearly as great. 